Birch-Andersen, A. (1955). J. gen. Microbiol. 13, 327-329

\title{
Reconstruction of the Nuclear Sites of Salmonella typhimurium from Electron Micrographs of Serial Sections
}

\author{
BY A. BIRCH-ANDERSEN \\ Statens Seruminstitut, Copenhagen, Denmark
}

SUMMARY: A method for obtaining a composite picture of the organisms from electron micrographs of serial sections is described. Evidence is presented that the same general structures are revealed, as far as the nuclear sites are concerned, by selective staining for deoxyribonucleic acid and by electron microscopy of sections.

Studies of electron micrographs of sections of cells fixed at various times during synchronous division do not permit the identification of characteristic configurations which suggest successive stages of nuclear division (BirchAndersen, Lark, Maaløe \& Sjöstrand, 1954). The probable reason for this is that the integrated picture of the cell is lost when individual thin sections are studied. In principle, this difficulty has been overcome by a method now developed for obtaining serial sections from which a composite picture of the cell can be constructed. However, the labour involved in this process makes the study and classification of large numbers of cells impracticable, and we have therefore investigated whether the nuclear sites, as they appear in composite pictures, may be identified as belonging to one of the configurations (dot, wedge, bar or dumbbell) described in the preceding paper (Lark, Maaløe \& Rostock, 1955). Preliminary studies now to be described indicate that this is possible, and that the state of a cell with respect to nuclear division may be judged from the shape of the nuclear vacuoles. If this be substantiated, a study of the structural details of the dividing nucleus by means of electron microscopy of thin sections may be undertaken.

\section{METHODS}

Fixation, dehydration, embedding and sectioning were performed as described by Birch-Andersen, Maaløe \& Sjöstrand (1953); the procedure for obtaining serial sections of bacteria by means of the Sjöstrand ultramicrotome will be described in a later publication. Electron micrographs were taken with the Philips E.M. 100 electron microscope equipped with the new $25 \mathrm{~A}$. objective lens. The standard objective aperture, as delivered by the factory, was used. Exposures were made at $60 \mathrm{kV}$., with a primary magnification of about $\times 4300$ on 35 mm. film (Kodak Fine Grain Positive Film), using a low beam intensity and without removing the embedding material.

For the present investigation some thirty organisms were selected, from each of which a complete series of longitudinal sections had been obtained. Com- 
posite pictures of each of these cells were made as follows. A series of positive contact prints was made on a $35 \mathrm{~mm}$. film, and the exposure time needed for producing five times enlarged negative prints on paper determined. Usually, the first as well as the last section in a series show no traces of nuclear sites in the bacterial cytoplasm; such sections were omitted in the rest of the procedure. This consisted of printing the remaining sections, one on top of the other, on to the same piece of photographic paper. The exposure time for each section was made equal to the predetermined time divided by the number of sections. So far no attempt has been made to estimate the thickness of the individual sections and to adjust exposure times accordingly. Between each exposure the outline of the cell was traced to permit the outline of the next section to be fitted to that of its predecessor. By this procedure we end with composite pictures of the kind shown on Pls. $1 \& 2$, which also show the series of individual sections, printed in positive, from which the reconstructions were made.

\section{RESULTS}

In the composite picture, printed in negative, the nuclear sites appear as black 'vacuoles' whose contents, as well as the bacterial cytoplasm, are white. A striking feature of the composite pictures is that, despite the irregularity in shape and distribution of the light areas in some of the individual sections, the reconstructions show that these areas combine to form definite nuclear regions ( 2,3 or $4 /$ cell) which seem not to communicate. The reconstructions presented here show nuclear regions which resemble the configurations designated by Lark et al. (1955) 'dot', 'wedge' and 'dumbbell' (Pl. 1, fig. $f$ 'dot'; Pl. 2, fig. $f$ 'wedge' and 'dumbbell'). The relatively high resolution in the composite pictures accounts for the fact that the outlines of the nuclear sites appear more irregular in the electron micrographs than in the photographs of stained cells. By means of the composite picture technique it has been possible to identify the types of nuclear sites shown in Pl. 1 and 2, and the bar type, described by Lark et al. (1955), has also been seen in some cases. It should be emphasized that about $30 \%$ of the nuclear sites so far examined by this technique could not be classified as belonging to one of the four configurations mentioned; however, these uncertain cases did not include configurations which could not be considered as intermediate types. As might be expected, in the composite pictures the most uncertain distinction is that between the dot and the wedge configurations.

The observations that the 'holes' or 'vacuoles' seen in individual sections combine to form closed regions and that the outlines of these regions resemble the nuclei as they appear in preparations stained selectively for deoxyribonucleic acid (DNA) strongly suggest that the two methods reveal the same general structures. It is not clear, however, whether the stained areas in Pls. 1 and 2 of the paper by Lark et al. (1955) correspond to the entire nuclear region as seen in the composite pictures of this paper or to the dense material inside these regions. It cannot be excluded, at the present time, that the fixation and dehydration processes which precede embedding, cause the DNA 
Journal of General Microbiology, Vol. 13, No. 2
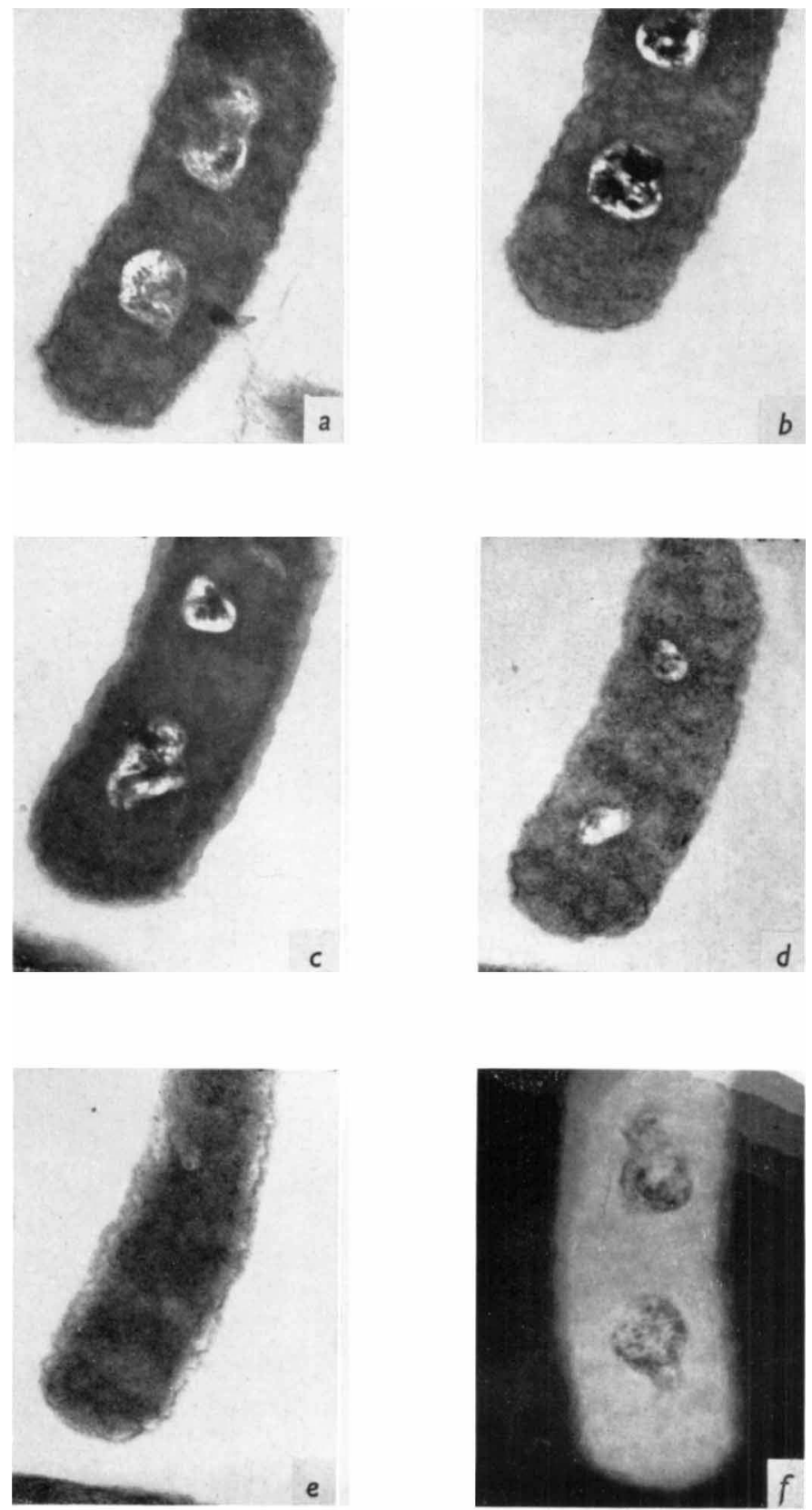

A Birch-Andersen-Reconstructions from electron microgiraphs. Plate 1 
Journal of General Microbiology, Vol. 13, No. 2
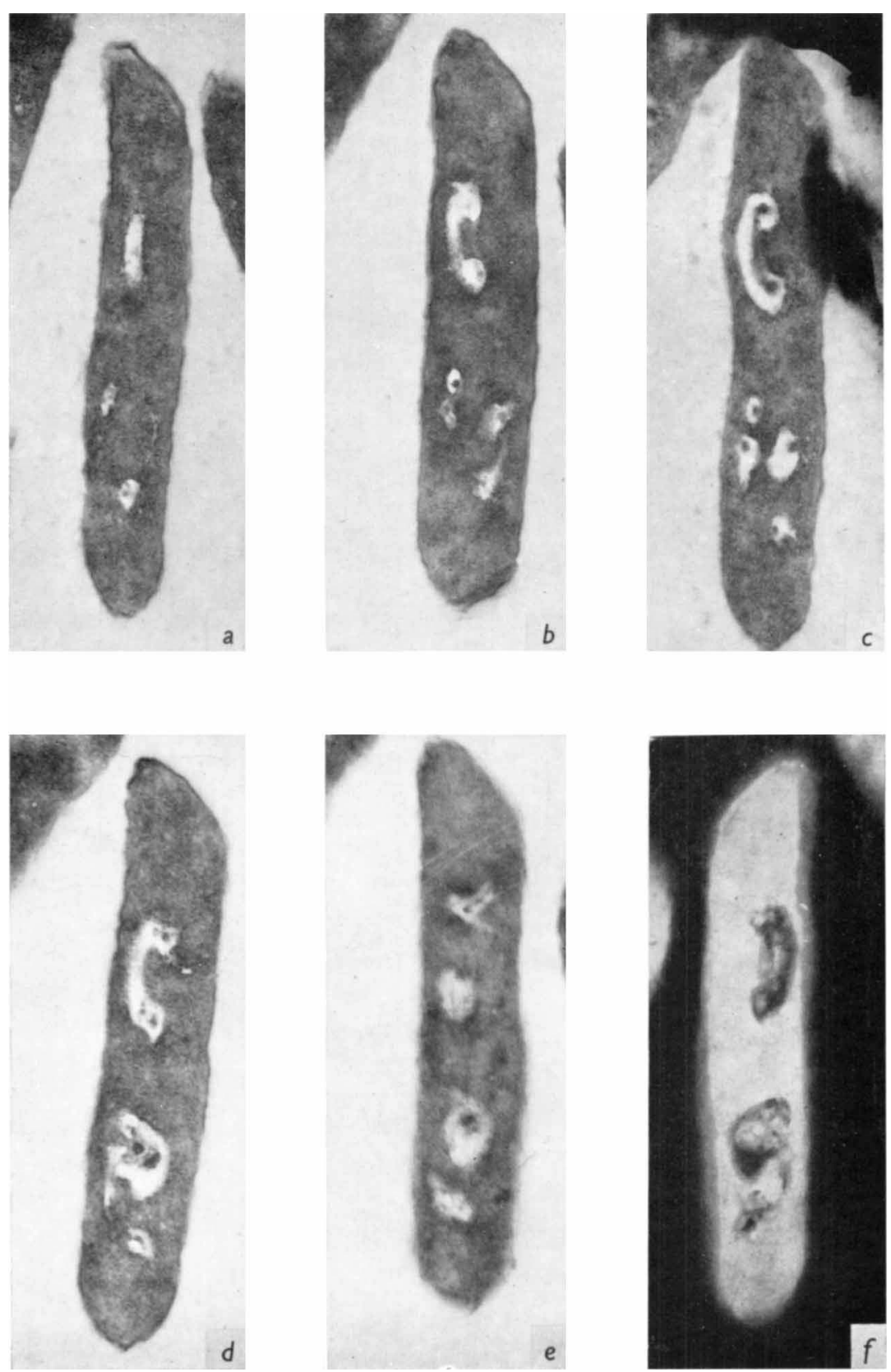

A. Birch-Andersen-Reconstructions hrom electron micrographs. Plate 2 
contained in the nuclear regions to shrink, or that acid hydrolysis and staining cause swelling of the DNA to make it fill the nuclear vacuoles more or less completely. For future studies of the structural details of the dividing nucleus it is, of course, essential to establish whether such artefacts are introduced during the preparation of the specimens. Control experiments comprising different fixation conditions and combination of staining and sectioning procedures, now being carried out in this laboratory, may settle these questions.

Special thanks are due to Mrs $H$. Ravn for valuable assistance in working out technical details of the procedure for collecting serial sections, and to Miss A.-G. Overgaard for carrying out the difficult photographic work involved in the making of the composite pictures.

\section{REFERENCES}

Birch-Andersen, A., Maaloe, O. \& Suöstrand, F. S. (1953). High resolution electron micrographs of sections of $E$. coli. Biochim. Biophys. Acta, 12, 395.

Birch-Andersen, A., Lark, K. G., Maaløe, O. \& SJöstrand, F. S. (1954). A study of sections of bacteria undergoing synchronous nuclear division. Proc. Int. Conf. Electron Microscopy. London, paper no. 72.

Lark, K. G., Maaløe, O. \& Rostock, O. (1955). Cytological studies of nuclear division in Salmonella typhimurium. J. gen. Microbiol. 13, 318.

\section{EXPLANATION OF PLATES}

\section{Plate 1}

Electron micrographs of individual sections of a Salmonella typhimurium cell (figs. $a-e$ ), and a composite picture (fig. $f$ ) produced as described on p. 328. Note that fig. $f$ is a negative and, therefore, a mirror image of the individual sections. Section $e$ was not used in making the composite picture, since no 'nuclear vacuoles' have been sectioned in this case. Magnification, $\times 21,500$.

\section{Plate 2}

Individual sections and composite picture, as on PI. 1. Here all sections were used to make the picture of fig. $f$. Magnification, $\times 21,500$. 